\title{
5G Antenna Array with Wide-Angle Beam Steering and Dual Linear Polarizations
}

\author{
Kirill Klionovski, Atif Shamim \\ Integrated Microwave Packaging Antennas and Circuits \\ Technology Laboratory \\ King Abdullah University of Science and Technology \\ (KAUST) \\ Thuwal, Kingdom of Saudi Arabia \\ kirill.klionovski@kaust.edu.sa, atif.shamim@kaust.edu.sa
}

\author{
Mohammad Said Sharawi \\ Electrical Engineering Department \\ King Fahd University of Petroleum and Minerals \\ (KFUPM) \\ Dhahran, Kingdom of Saudi Arabia \\ msharawi@kfupm.edu.sa
}

\begin{abstract}
In this paper, we present the design of a switchedbeam antenna array at millimeter-wave frequencies for future 5G applications. The proposed antenna array is based on wideband patch antenna elements and a Butler matrix feed network. The patch antenna has a broad radiation pattern for wide-angle beam steering and allows the simultaneous operation with two orthogonal linear polarizations. A combination of two separated Butler matrices provides independent beam steering for both polarizations in the wide operating band. The antenna array has a simple multilayer construction, and it is made on a low-cost Rogers laminate.
\end{abstract}

Keywords-millimeter-wave antenna array; 5 G antennas; Butler matrix; wideband patch antenna; switched-beam antenna.

\section{INTRODUCTION}

New millimeter-wave and 5G wireless standards are being proposed as a solution to the increasing demand for more bandwidth and higher data transfer rates. These high data rate goals can be facilitated by wideband antenna arrays with wideangle beam steering, which can operate with two orthogonal linear polarizations [1]. The two polarizations can enhance the capacity and thus can boost the spectral efficiency of $5 \mathrm{G}$ communication links. Beam steering can be achieved by two ways, (1) by the use of phase shifters for each element of an array, or (2) by different types of beamforming networks, such as Butler matrix, Blass matrix, Nolen matrix, Rotman lens, etc. Phase shifters for millimeter-wave frequencies are quite expensive, narrow-band, and lossy [2]. A beamforming network, on the other hand, can avoid these disadvantages. Butler matrix is a widely-used beamforming network, which consists of standard elements: 3-dB directional couplers, crossovers and delay lines. A patch antenna can be used as a wideband element in an antenna array with broad radiation pattern. Some designs of a single polarization, millimeter-wave antenna arrays with Butler matrix feed network have been described in [3-5].

In the present paper, we investigate the design of a wideband patch antenna array with a Butler matrix feed network for the $5 \mathrm{G}$ frequency band. We present simulated and measured voltage standing wave ratio (VSWR) and radiation patterns for a wide- band patch antenna, and simulated VSWR and radiation patterns for an antenna array.

\section{Design of The Elements of The ARray}

Radiating elements for a $5 \mathrm{G}$ antenna array have to provide wideband and wide radiation patterns in $\mathrm{E}$ - and $\mathrm{H}$-plane, as well as it is required to operate with two linear polarizations. To obtain a broad radiation pattern and a wide frequency band, we use a stacked patch antenna design [6]. The patch has two dielectric layers of the Rogers RO3003 laminate with a permittivity of 3, a thickness of $0.5 \mathrm{~mm}$ and a radius of $3.8 \mathrm{~mm}$ for each layer. Each layer has a square metal plate with angled circular cutouts. The lengths of the bottom and the top squares are 2.85 and $2.65 \mathrm{~mm}$ respectively. The radius of all cutouts is $0.95 \mathrm{~mm}$. The patch is excited by a $50-\Omega$ coaxial cable, which is located $0.78 \mathrm{~mm}$ from the axis of the patch antenna. The central core of the coaxial cable has electrical contact with only the bottom plate. The design of the wideband stacked patch antenna (shown in Fig. 1) has been fabricated and measured.

Figs. 2 and 3 show the measured and simulated radiation patterns at the frequency of $30 \mathrm{GHz}$ and VSWR of the patch antenna. In Fig. 2, the angle $\theta$ is measured from the normal to the antenna plane. Fig. 2 shows that the $3 \mathrm{~dB}$ beam-width for the patch antenna is $90^{\circ}$. Fig. 3 shows that VSWR of the patch antenna is less than 2 for the frequencies $27.2-35.3 \mathrm{GHz}$. This design of the patch antenna can be easily realized to operate with two orthogonal polarizations by using an excitation of the bottom plate by two coaxial cables, which are located at the angle of 90 degrees about the axis of the patch antenna.
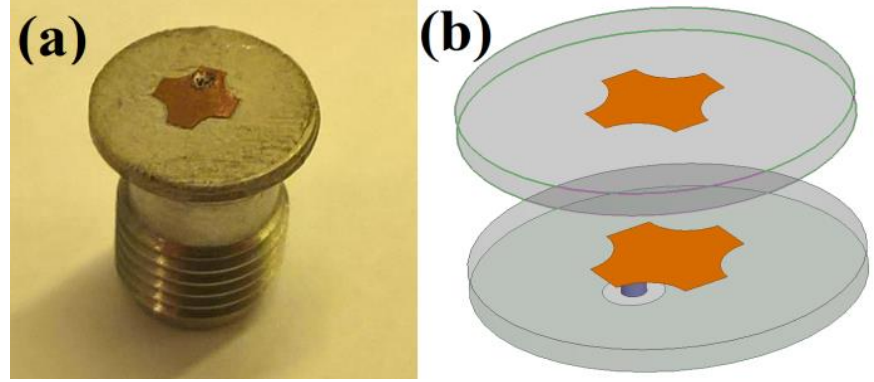

Fig. 1. (a) Photo of the fabricated patch antenna and (b) a view of the two separate layers of the antenna. 


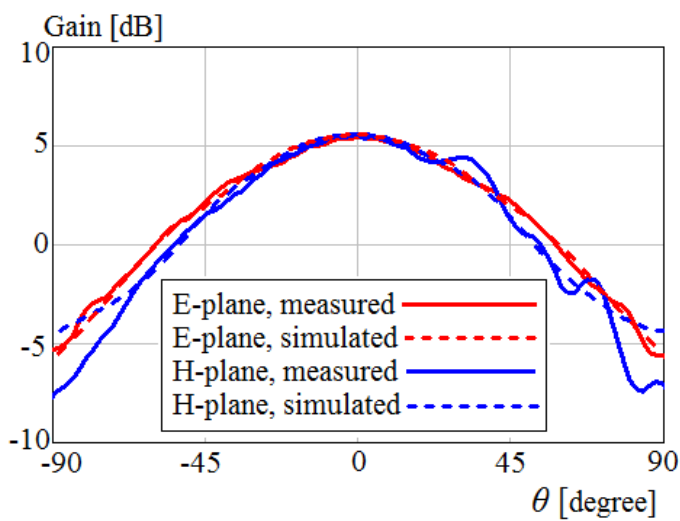

Fig. 2. Radiation patterns of the patch antenna.

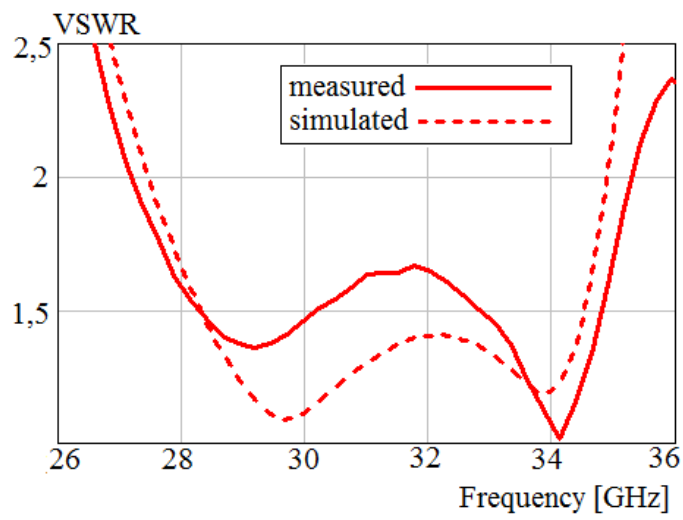

Fig. 3. VSWR of the patch antenna.

\section{Design of The ANTENna ARray}

The geometry of a 16-elements antenna array is shown in Fig. 4. The Butler matrix feed network is based on the Rogers RO3003 laminate with a thickness of $0.13 \mathrm{~mm}$. The Butler matrix includes four $3-\mathrm{dB}$ quadrature directional couplers, two crossovers, and two delay lines. Figs. 5, 6 plot the simulated VSWR and radiation patterns at the frequency of $28.5 \mathrm{GHz}$ for the antenna array. Fig. 5 shows that VSWR of the antenna is less than 2 for the frequencies 27-33.7 GHz. Isolation between the feed ports is more than $-15 \mathrm{~dB}$. Fig. 6 shows radiation patterns of the array when different ports are excited. The patterns plot in the plane, which is perpendicular to the plane of

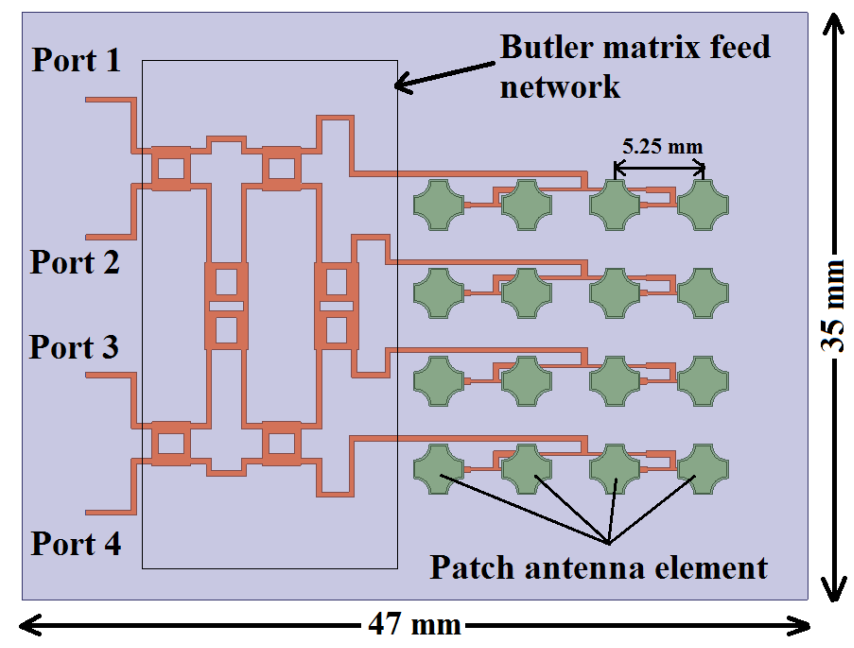

Fig. 4. The design of the 16 -elements antenna array.

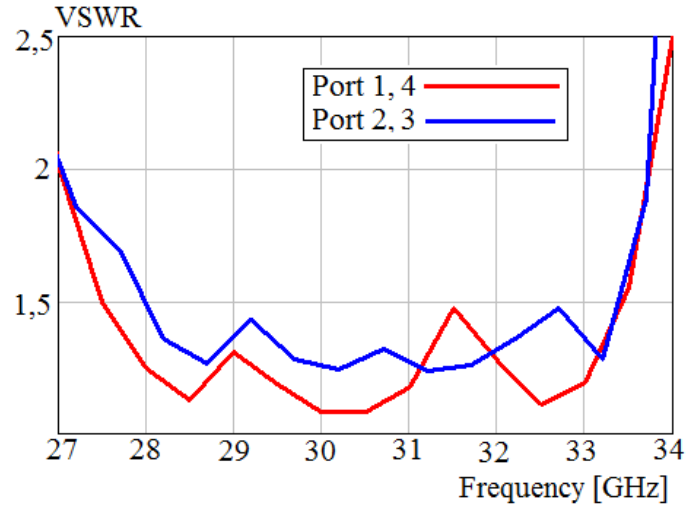

Fig. 5. VSWR of the antenna array.

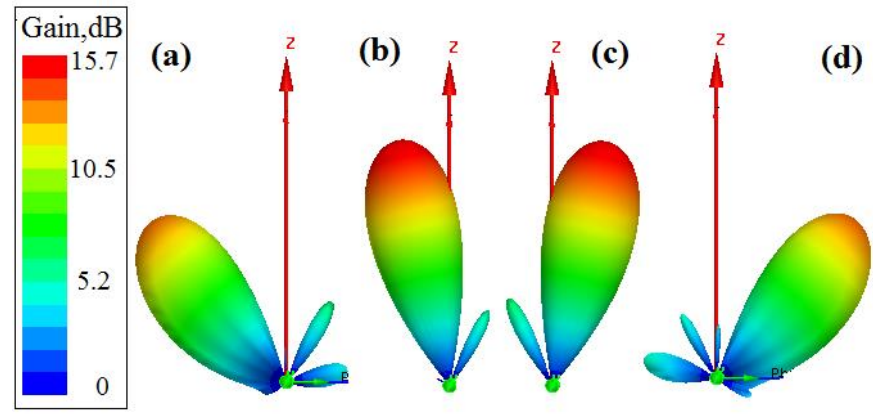

Fig. 6. Radiation patterns of the antenna array with different port excitation: port 3 (a), port 1 (b), port 4 (c), port 2 (d).

the array. The directions of the main beams are $-42^{\circ},-13^{\circ}, 13^{\circ}$ and $42^{\circ}$ when port $3,1,4$ and 2 is excited, respectively. The proposed design of the Butler matrix allows obtaining wide impedance bandwidth. However, bandwidth for stable beam steering needs to be further optimized.

\section{CONCLUSIONS}

A planar low-cost switched-beam antenna array for $5 \mathrm{G}$ telecommunications is proposed. The array is based on a Butler matrix feed network with wideband patch antenna elements. It operates in the frequency range of $27-33.7 \mathrm{GHz}$ and provides wide-angle beam steering with two orthogonal polarizations.

\section{REFERENCES}

[1] Fa-Long Luo and Charlie Zhang, Signal Processing for 5G: Algorithms and Implementations. New Jersey: John Wiley and Sons, 2016.

[2] I. Uchendu and J. Kelly, "Survey of Beam Steering Techniques Available for Millimeter Wave Applications," PIER, vol. 68, pp. 35-64, 2016.

[3] C. H. Tesng, C. J. Chen, and T. H. Chu, "A low-cost 60-GHz switchedbeam patch antenna array with Butler matrix network," IEEE Antennas Wireless Propag. Lett., no. 7, pp. 432-435, July 2008.

[4] W. F. Moulder, W. Khalil, and J. L. Volakis, "60-GHz TwoDimensionally Scanning Array Employing Wideband Planar Switched Beam Network," Antennas Wireless Propag. Lett., vol. 9, pp. 818-821, 2010.

[5] A. T. Alreshaid, M. S. Sharawi, S. Podilchak, and K. Sarabandi, "Compact millimeter-wave switched-beam antenna arrays for short range communications," Microwave and Optical Technology Letters, vol. 58, no. 8, pp. 1917-1921, Aug. 2016.

[6] V. V. Golovin and Y. N. Tyschuk, "Multielement patch antenna array of operated polarization of Ku-band," in Proc. 9th Int. Conf. on Antenna Theory and Techniques (ICATT), Odessa, Ukraine, 2013, pp. 286-288. 